\title{
Nonlinear moment matching-based model order reduction
}

\author{
Tudor C. Ionescu, Alessandro Astolfi, Fellow, IEEE
}

\begin{abstract}
In this paper we present a time-domain notion of moments for a class of single-input, single-output nonlinear systems in terms of the evolution of the output of a generalized signal generator driven by the nonlinear system. We also define a new notion of moment matching and present a family of (nonlinear) parametrized reduced order models that achieve moment matching. We establish relations with existing notions of moment for nonlinear systems, showing that the newly derived and the existing families of reduced order models that achieve nonlinear moment matching, respectively, are equivalent. Furthermore, we compute the reduced order model that matches the moments at two chosen signal generators (one exciting the input of the system and another driven by the system), simultaneously. We also present a family of models computed on the basis of a nonlinear extension of the Petrov-Galerkin projection that achieve moment matching. Finally, we specialise the results to the case of nonlinear, input-affine systems.
\end{abstract}

Index Terms-Nonlinear moment matching, signal generator, family of parametrized reduced order models, two-sided, interconnection.

\section{INTRODUCTION}

$\mathbf{T}$ HE MODEL REDUCTION PROBLEM for linear and nonlinear systems has been widely studied in the systems and control community. Since the modelling of processes and phenomena leads to models consisting of a large number of differential equations, the resulting systems are not always suitable for analysis and control design. Hence, this problem is of great importance in applications, because its solution yields reduced order models (i.e., models of reduced complexity) that can be used in practice. In the problem of model reduction moment matching techniques represent an efficient tool, see e.g., [1] for a complete overview for linear systems. In such techniques the (reduced order) model is obtained by constructing a lower degree rational function that approximates the given transfer function (assumed rational). The classical notion of moment has been defined in [1], based on the series expansion of the transfer function of the linear system (see also [2]-[4]). The low degree rational function matches (some of) the terms of the original transfer function at various points in the complex plane. However, since the calculation of moments is costly, the reduced order models that match a

T. C. Ionescu is with the Dept. of Automatic Control \& Systems Engineering, Politehnica Univ. of Bucharest, 060042 Bucharest, Romania and with the "Gheorghe Mihoc-Caius Iacob" Institute of Mathematical Statistics and Applied Mathematics of the Romanian Academy, 050711 Bucharest, Romania. E-mail: td.c.ionesculgmail.com.

A. Astolfi is with the Department of Electrical and Electronic Engineering, Imperial College London, SW7 2AZ, London, UK and with Dipartimento di Informatica, Sistemi e Produzione, Università di Roma Tor Vergata, 00133 Rome, Italy. E-mail: a.astolfi@imperial.ac.uk.

This work has been supported by the EPSRC grant "Control for Energy and Sustainability", reference EP/G066477/1. prescribed number of moments are computed efficiently using Krylov projection techniques, which do not require the direct computation of the moments (see, e.g., [5]-[7]).

Recently, a time-domain notion of moment for linear and nonlinear systems has been proposed in [8], [9]. We give a brief overview of the arguments developed in [8]. The problem is formulated as follows. Given a nonlinear system of dimension $n$

$$
\dot{x}=f(x, u), y=h(x),
$$

where $x(t) \in \mathbb{R}^{n}, u(t) \in \mathbb{R}, y(t) \in \mathbb{R}$ and $f$ and $h$ are smooth mappings, such that $f(0,0)=0$ and $h(0)=0$, find a reduced order model of dimension $\nu<n$

$$
\dot{\xi}=\varphi(\xi, u), \eta=\psi(\xi),
$$

with $\xi(t) \in \mathbb{R}^{\nu}$, that approximates the system (1) based on a moment matching criterion. The solution in [8] has been developed as follows. First, the notion of moment of a nonlinear system has been defined in relation to the (welldefined steady-state) evolution of the output of the nonlinear system driven by a chosen input yielded by a signal generator

$$
\dot{\omega}=s(\omega), \theta=l(\omega),
$$

with $\omega(t) \in \mathbb{R}^{\nu}, \theta(t) \in \mathbb{R}$ and $s$ and $l$ smooth mappings, such that $s(0)=0$ and $l(0)=0$, as depicted in Figure 1. The moments are related to the solution of a nonlinear

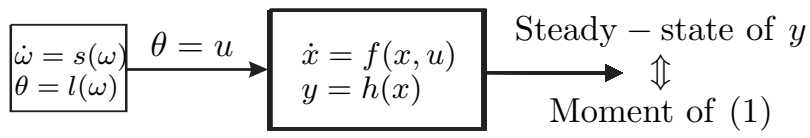

Fig. 1. Diagram illustrating the definition of moment

(Sylvester-like) partial differential equation (see [10], [11] for the linear arguments). Using similar arguments, the moment of (2) is related to the steady-state evolution of the output of the system when excited by the signal generated by (3). Moment matching is achieved when the moment of the system (2) is equal to the moment of (1). Based on this property, a family of parametrized, reduced order models that achieve moment matching has been computed. The free parameters can be used to enforce desired properties on the reduced order model, such as stability and passivity (see, e.g., [12], [13]). For linear systems, in [14], this approach has yielded a new family of parametrized reduced order models achieving moment matching and the equivalence to the Krylov projection-based models has been established. Furthermore, the problem of matching a number of moments larger than the dimension of the reduced order model has been studied in, e.g., [14], where an approach 
based on interconnection of low order approximations has been taken and in [15], where the problem of interpolating $(\rho+1) \nu$ moments with a time-delay system of order $\nu$ with $\rho$ delays has been considered.

In this paper we present a new time-domain notion of moment for a class of single-input, single-output nonlinear systems, in terms of the evolution of the output of the "swapped interconnection" of the system with a (generalised) signal generator. The term "swapped" refers to the interconnection between the nonlinear system (1) and a signal generator, where the nonlinear system (1) drives the signal generator, see Figure 2. The new notion of moment is defined based on

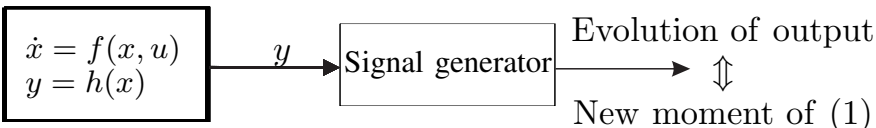

Fig. 2. Diagram illustrating the proposed definition of moment for the nonlinear system (1) based on the "swapped" interconnection between the system and the signal generator

the evolution of the output of the interconnection depicted in Figure 2. Similar to [8], this notion of moment is related to the (unique) solution of a nonlinear partial-differential equation. This is an extension of the linear arguments developed in [9], [14]. Under some technical assumptions, the moment of the given nonlinear system is defined in a one-to-one relation with the evolution of the output of such interconnection. However, unlike the linear case the proposed PDE that characterises the notion of moment is not dual to the Sylvester-like nonlinear PDE from [8], hence, throughout the rest of the paper, when required, we use the terminology "swapped interconnection" rather than "dual". The nonlinear system (2) is said to match the given moment if the interconnection with the generalised signal generator preserves the structure of the moment and yields an output that matches the evolution of the output of the same generalised signal generator driven by the given nonlinear system, see Figure 3. Hence, we compute a new

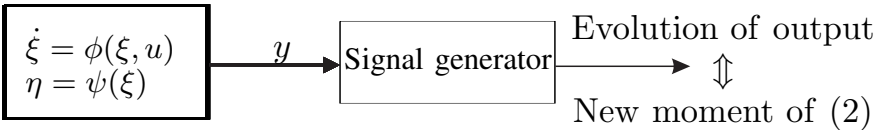

Fig. 3. Diagram illustrating the proposed definition of moment for the reduced order model (2) based on the "swapped" interconnection between the system and the signal generator

parametrized family of reduced order models that achieve moment matching in the aforementioned sense. Since, in practice, stable models are of significant interest (e.g., for power systems, where transients are of importance, see [16], [17]), we show that the sub-family of stable models that achieve moment matching can be computed using a classical linearisation argument. Furthermore, we prove that the notion of moment matching based on the "swapped interconnection" is equivalent to the notion of moment matching described in [8], i.e., any model from one family of models that match the moments of the system can be found in the "swapped" family of models. We also address the problem of "two-sided" moment matching for nonlinear systems, i.e., we compute the reduced order models that simultaneously match the moment in the sense of [8] and the moment in the sense of the "swapped interconnection" approach. The result is a nonlinear extension of the computation of a reduced order, linear model that matches a number of moments equal to twice its order, based on employing "two-sided" Krylov projections, see, e.g., [5], [18], [19]. We also present the relation with the models obtained using a nonlinear extension of the Petrov-Galerkin projection method, as described in [20]. Furthermore, since in practice most of the models stemming from applications are affine in the input, we present explicit results for this class of systems. Note that the results are general, since the number of assumptions made is minimal. Finally, although in this paper we study the single-input, single-output case, the results are directly applicable to the multiple-input, multiple-output case.

The paper is organized as follows. In Section II-A we give a short presentation of the notion of time-domain moments for nonlinear systems and the resulting family of reduced order models that achieve moment matching. In Section II-B we briefly overview a nonlinear extension of the PetrovGalerkin projection method and present the resulting family of reduced order models that achieve moment matching in the sense of Section II-A. In Section III-A we define and analyse the output of a generalized nonlinear signal generator driven by the system. The resulting structure of the dynamics of the interconnection provides the definition of a new notion of moment. We define moment matching in terms of finding a low order system that preserves the structure of the interconnection with the signal generator. We compute a family of parametrized, reduced order, nonlinear models that achieve moment matching in the aforementioned sense. We establish a relation between the notion of moment and moment matching based on the output of the system driven by a signal generator and its "swapped" counterpart, through a necessary and sufficient condition. In Section III-B we briefly discuss the problem of moment matching-based model order reduction, as in Section III-A, such that the approximant has an asymptotically stable equilibrium. In Section III-C we compute the reduced order models that simultaneously match the moment of the given nonlinear system in the sense of [8] and the moment of the nonlinear system in the sense of Section III-A. The nonlinear Petrov-Galerkin projection-based extension of the results from Section III-C is given in Section III-D. In Section IV we apply the results developed in Section III to the case of nonlinear systems, affine in the input. Section $\mathrm{V}$ contains an example which illustrates the theory. The paper is completed by some concluding remarks.

Preliminary results of this work may be found in [21] and [20], where proofs have not been given and the developments were made on particular cases.

Notation: $\mathbb{R}$ is the set of real numbers and $\mathbb{C}$ is the set of complex numbers. $\mathbb{R}^{+}$denotes the set of positive real numbers and $\mathbb{R}^{-}$is the set of negative real numbers. $\mathbb{C}^{0}$ is the set of complex numbers with zero real part and $\mathbb{C}^{-}$denotes the set of complex numbers with negative real part. $M^{*} \in \mathbb{C}^{n \times m}$ is the complex-conjugate transpose of the matrix $M \in \mathbb{C}^{m \times n}$. If $M \in \mathbb{R}^{m \times n}$, then $M^{*}=M^{T}$ is the transpose of the matrix M. $\sigma(A)$ denotes the set of eigenvalues of the square matrix 
$A$ and $\emptyset$ denotes the empty set. Let $x \in \mathbb{R}^{n}$ and $f: \mathbb{R}^{n} \rightarrow \mathbb{R}^{m}$ be a differentiable function, then $\left.\frac{\partial f(x)}{\partial x}\right|_{x=a} \in \mathbb{R}^{m \times n}$ denotes the Jacobian of $f$ evaluated at $a \in \mathbb{R}^{n}$.

\section{Preliminaries}

\section{A. Nonlinear moment matching - system driven by a signal generator}

Consider the single-input, single-output, nonlinear system described by equations (1) and the signal generator (3). Consider the interconnected system

$$
\dot{\omega}=s(\omega), \dot{x}=f(x, l(\omega)), y=h(x) .
$$

Suppose that $f$ and $h$ are smooth mappings defined in the neighbourhood of the origin of $\mathbb{R}^{n}$ and $s$ and $l$ are smooth mappings defined in the neighbourhood of the origin of $\mathbb{R}^{\nu}$. Furthermore assume $f(0,0)=0, s(0)=0, l(0)=0$ and $h(0)=0$. Throughout the paper the results are local, although global versions are easy to give. The following definitions and assumptions have been given in [8].

Assumption 1: There exist a unique smooth mapping $\pi(\omega)$ locally defined in the neighbourhood of 0 , which is the solution of the partial differential equation

$$
\frac{\partial \pi(\omega)}{\partial \omega} s(\omega)=f(\pi(\omega), l(\omega))
$$

Assumption 1 implies that system (4) has an invariant manifold $x=\pi(\omega)$, with $\pi(0)=0$, on which the restricted dynamics of the system is described by (3).

Assumption 2: The signal generator (3) is observable ${ }^{1}$ and Poisson stable ${ }^{2}$.

Definition 1: [8], [23] Consider the system (1) and the signal generator (3). Assume that the signal generator (3) is observable and that the equilibrium $\omega=0$ of the system (3) is Poisson stable. Furthermore, assume that $\pi$ is the unique solution of (5). Then, we call the function $h \circ \pi$ the moment of (1) at $\{s, l\}$.

The assumptions and definitions above allow to derive a result relating the notion of moment of the nonlinear system (1) with the (well-defined) steady-state response (provided it exists) of the output of the interconnection (4).

Theorem 1: [8] Consider system (1) and the signal generator (3). Suppose Assumption 2 holds. Assume that the zero equilibrium of the system $\dot{x}=f(x, 0)$ is locally exponentially stable and $\omega(0) \neq 0$. Then Assumption 1 holds and the moment of (1) at $\{s, l\}$ coincides with the (locally well-defined)

\footnotetext{
${ }^{1}$ The system (3) is observable if for any pair of initial conditions $\omega_{a}(0) \neq$ $\omega_{b}(0)$, the corresponding output trajectories $l\left(\omega_{a}(t)\right)$ and $l\left(\omega_{b}(t)\right)$ are such that $l\left(\omega_{a}(t)\right)-l\left(\omega_{b}(t)\right) \neq 0$ for all $t$.

${ }^{2}$ An equilibrium point $\bar{\omega}$ is said to be Poisson stable if the trajectory $\omega(t)$, solution of the equation $\dot{\omega}=s(\omega)$, passes close to $\bar{\omega}$ for arbitrarily large times, in forward and backward direction. Hence, if every point in a neighbourhood of $\bar{\omega}$ is Poisson stable, no trajectory of (3) can decay to zero as time tends to infinity, see e.g., [22, Chapter 8].
}

steady-state response ${ }^{3}$ of the output of the interconnected system (1).

Now, we present the definition of a system that matches the moment $h \circ \pi$ of (1) at $\{s, l\}$.

Definition 2: [8] The system (2), with $\xi(t) \in \mathbb{R}^{\nu}$, matches the moment of (1) at $\{s, l\}$ if it has the same moment at $\{s, l\}$ as (1), i.e., the equation

$$
\varphi(p(\omega), l(\omega))=\frac{\partial p(\omega)}{\partial \omega} s(\omega)
$$

has a unique solution $p$, locally defined in the neighbourhood of the origin, such that

$$
h(\pi(\omega))=\psi(p(\omega)),
$$

where $\pi$ is such that Assumption 1 holds.

To compute the reduced order models that achieve moment matching we make the following assumption.

Assumption 3: The mappings $\psi$ and $p$ are smooth and such that $\psi(0)=0$ and $p(0)=0$. Furthermore (6) holds and $p$ possesses a local inverse.

Note that Assumption 3 holds for $p(\omega)=\omega$ and $\psi(\omega)=$ $h(\pi(\omega))$. Hence, provided Assumptions 1-3 hold, a family of reduced order models, all achieving moment matching at $\{s, l\}$, is described by

$$
\Sigma_{\delta(\xi)}:\left\{\begin{array}{l}
\dot{\xi}=s(\xi)-\delta(\xi) l(\xi)+\delta(\xi) u, \\
\psi=h(\pi(\xi))
\end{array}\right.
$$

with $\xi(t) \in \mathbb{R}^{\nu}$, where $\delta$ is such that the equation

$$
s(p(\omega))+\delta(p(\omega)) l(\omega)-\delta(p(\omega)) l(p(\omega))=\frac{\partial p(\omega)}{\partial \omega} s(\omega)
$$

has the unique solution $p(\omega)=\omega$.

\section{B. Nonlinear Petrov-Galerkin projection-based moment matching}

In this section we present a new family of reduced order models that achieve moment matching, built through a nonlinear extension of the Petrov-Galerkin projection method in which the projector is given by the mapping

$$
\varrho: \mathbb{R}^{n} \rightarrow \mathbb{R}^{n}, \varrho(\pi(\xi))=\xi .
$$

In the linear case, $\pi$ and $\varrho$ are $\pi(\xi)=V \xi$ and $\varrho(x)=W^{*} x$, respectively, and equation (10) becomes $W^{*} V=I$. The following result presents the family of nonlinear Petrov-Galerkin projection-based reduced order models that achieve moment matching at $\{s, l\}$.

${ }^{3} \mathrm{We}$ use the notion of steady-state response as described in [24], see also [25]. In lay terms, the steady-state response of a system fed by some specific input is a function of time to which the actual response "converges" as time increases, provided such convergence exists. In detail, let $x(t, x(0), u)$ denote the value of the state of (1) at time $t$ starting from the initial condition $x(0)$ under the effect of $u$. Suppose there exists $x_{\mathrm{ss}}(0)$ such that $\lim _{t \rightarrow \infty}\left\|x(t, x(0), u)-x\left(t, x_{\mathrm{ss}}(0), u\right)\right\|=0$, for all $x(0)$. Then the response $x_{\mathrm{ss}}(t)=x\left(t, x_{\mathrm{ss}}(0), u\right)$ is the steady-state of the system (1). Hence, the steady-state response of (1) is $y_{\mathrm{ss}}(t)=h\left(x_{\mathrm{ss}}(t)\right)$. 
Theorem 2: Consider system (1) and the signal generator (3) assumed observable. Suppose $\pi$ is the unique solution of equation (5). Consider the family of models

$$
\begin{aligned}
& \dot{\xi}=\left.\frac{\partial \varrho(x)}{\partial x} f(x, u)\right|_{x=\pi(\xi)}, \\
& \psi=h(\pi(\xi)),
\end{aligned}
$$

where $\varrho$ is such that equation (10) holds. Assume that the zero equilibrium of (11) is exponentially stable. Then all models in the family (11) match the moment of system (1) at $\{s, l\}$.

Proof: To prove the claim it is sufficient to show that the moment at $\{s, l\}$ of all models (11) coincides with the moment of (1). For, consider the partial differential equation

$$
\left.\frac{\partial \varrho(x)}{\partial x} f(x, l(\omega))\right|_{x=\pi(p(\omega))}=\frac{\partial p(\omega)}{\partial \omega} s(\omega)
$$

and note that, by equation (5), and the property $\left.\frac{\partial \varrho(x)}{\partial x}\right|_{x=\pi(\xi)} \frac{\partial \pi(\xi)}{\partial \xi}=I$, resulting from (10), the function $p(\omega)=\omega$ is a solution of (12). By the centre manifold theory, this solution is unique, see [26]. As a result, The moment at $\{s, l\}$ of all models (11) is given by $h(\pi(\omega))$, which proves the claim.

Note that in the linear case a reduced order model of the linear system $\dot{x}=A x+B u, y=C x$ is obtained by using the projection $x=V \xi$, i.e, the reduced order model is given by $\dot{\xi}=W^{*} A V \xi+W^{*} B u, \psi=C V \xi$. By [1, Chapter 11], if $V$ and/or $W$ are the Krylov projections, then the reduced order model is an approximation of the linear system that matches a prescribed set of moments.

\section{NONLINEAR MOMENT MATCHING, A NEW APPROACH -} THE SIGNAL GENERATOR DRIVEN BY THE SYSTEM

\section{A. Main results}

In this section we present a new general framework for nonlinear moment matching based on "swapping" the interconnection between the system and the (generalized) signal generator.

Consider the generalized signal generator defined by the equations

$$
\begin{aligned}
\dot{\varpi} & =q(\varpi, v), \varpi(0)=0, \\
d & =\boldsymbol{v}(\varpi, x),
\end{aligned}
$$

with $\varpi(t) \in \mathbb{R}^{\nu}, d(t) \in \mathbb{R}^{\nu}, q: \mathbb{R}^{\nu} \times \mathbb{R} \rightarrow \mathbb{R}^{\nu}$ a smooth mapping, with $q(0,0)=0$ and $v: \mathbb{R}^{\nu} \times \mathbb{R}^{n} \rightarrow \mathbb{R}^{\nu}$ a smooth mapping, with $\boldsymbol{v}(0,0)=0$ and $\frac{\partial \boldsymbol{v}(\varpi, x)}{\partial \varpi}$ full rank around $(0,0)$. Furthermore, assume that there exists $\rho: \mathbb{R}^{n} \rightarrow \mathbb{R}^{\nu}$ such that, locally, $\boldsymbol{v}(\rho(x), x)=0$, i.e., $d$ restricted to the manifold $\varpi=\rho(x)$ is zero. Consider the interconnection between the nonlinear system (1) and the signal generator (13), described by the relation $v=y$, as depicted in Figure 4, i.e., given by

$$
\begin{aligned}
\dot{x} & =f(x, u), \\
\dot{\omega} & =q(\varpi, h(x)), \\
d & =\boldsymbol{v}(\varpi, x) .
\end{aligned}
$$

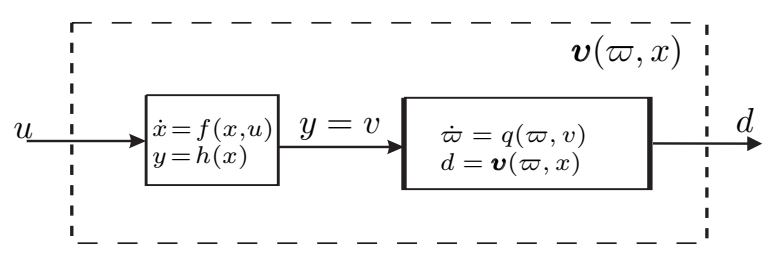

Fig. 4. Diagram describing the signal $d(t)$.

The following result describes the evolution of the signal $d$, restricted to the manifold $\varpi=\rho(x)$, based on the solution of a nonlinear Sylvester-like partial differential equation.

Lemma 1: Consider the nonlinear signal generator (13). Then $d(0)=0$ and $u(t)=0$ for all $t \geq 0$ imply $d(t)=0$ for all $t$, if and only if the nonlinear "Sylvester-like" partial differential equation

$$
\left.\left(\frac{\partial \boldsymbol{v}(\varpi, x)}{\partial \varpi} q(\varpi-d, h(x))+\frac{\partial \boldsymbol{v}(\varpi, x)}{\partial x} f(x, 0)\right)\right|_{\varpi=\rho(x)}=0
$$

holds for all $x$ around zero.

Proof: Note that $\dot{d}=\dot{\boldsymbol{v}}(\varpi, x)=\frac{\partial \boldsymbol{v}}{\partial \varpi} \dot{\varpi}+\frac{\partial \boldsymbol{v}(\varpi, x)}{\partial x} \dot{x}=$ $\frac{\partial \boldsymbol{v}(\varpi, x)}{\partial \varpi} q(\varpi, h(x))+\frac{\partial \boldsymbol{v}(\varpi, x)}{\partial x} f(x, u)$. By the existence of the mapping $\rho$, this further yields

$$
\begin{aligned}
& \left.\dot{d}\right|_{\varpi=\rho(x)}=\left.\frac{\partial \boldsymbol{v}(\varpi, x)}{\partial \varpi}[q(\varpi, h(x))-q(\varpi-d, h(x))]\right|_{\varpi=\rho(x)} \\
& +\left.\frac{\partial \boldsymbol{v}(\varpi, x)}{\partial x}\right|_{\varpi=\rho(x)}[f(x, u)-f(x, 0)] \\
& +\left.\frac{\partial \boldsymbol{v}(\varpi, x)}{\partial \varpi} q(\varpi-d, h(x))\right|_{\varpi=\rho(x)}+\left.\frac{\partial \boldsymbol{v}(\varpi, x)}{\partial x}\right|_{\varpi=\rho(x)} f(x, 0) .
\end{aligned}
$$

Then

$$
\left.\dot{d}\right|_{\varpi=\rho(x), u=0}=0
$$

holds if and only if $v$ satisfies the equation

$$
\frac{\partial \boldsymbol{v}(\varpi, x)}{\partial \varpi} q(\varpi-d, h(x))+\frac{\partial \boldsymbol{v}(\varpi, x)}{\partial x} f(x, 0)=0,
$$

for $\varpi=\rho(x)$, yielding (15).

Assuming that (15) holds locally around zero, the nonlinear interconnected system (14) can be rewritten based on the evolution of the coordinates $x$ and $d$ as

$$
\begin{aligned}
\dot{x} & =f(x, u), \\
\dot{d} & =\frac{\partial \boldsymbol{v}(\varpi, x)}{\partial \varpi}[q(\varpi, h(x))-q(\varpi-d, h(x))] \\
& +\frac{\partial \boldsymbol{v}(\varpi, x)}{\partial x}[f(x, u)-f(x, 0)], \\
\vartheta & =d,
\end{aligned}
$$

with $\varpi=\rho(x)$. Note that $(18)$ is such that $(x, d)=(0,0)$ is an equilibrium for $u=0$. We are now ready to propose a new definition of moment for the nonlinear system (1). Throughout the rest of the paper we make the following standing assumption.

Assumption 4: The mapping $\boldsymbol{v}$ in (13b) is the unique solution of the nonlinear partial differential equation (15) and $\rho$ is a smooth mapping such that $\boldsymbol{v}(\rho(x), x)=0$. 
Definition 3: Consider the nonlinear system (1) and the generalized nonlinear signal generator (13). Suppose that Assumption 4 holds. We call the moment of (1) at $q$ the mapping

$$
\left.\frac{\partial \boldsymbol{v}(\varpi, x)}{\partial x}[f(x, u)-f(x, 0)]\right|_{\varpi=\rho(x)},
$$

locally defined in the neighbourhood of the origin, where $\rho$ is as in Assumption 4.

We now give the definition of a model that achieves moment matching. It is based on searching for the dynamics of the output of the generalised signal generator fed by the model that mimics the evolution of $d$ from (18). Consider the nonlinear system (2) given by

$$
\begin{aligned}
& \dot{\xi}=\varphi(\xi, u), \\
& \eta=\psi(\xi),
\end{aligned}
$$

where $\xi(t) \in \mathbb{R}^{\nu}, u(t) \in \mathbb{R}$ and $\eta(t) \in \mathbb{R}$ and $\varphi$ and $\psi$ are smooth mappings with $\varphi(0,0)=0$ and $\psi(0)=0$. Consider the interconnection $v(t)=\eta(t)$, between (2) and the signal generator defined by (13a) with the output $\zeta(t) \in \mathbb{R}^{\nu}$ (see Figure 5).

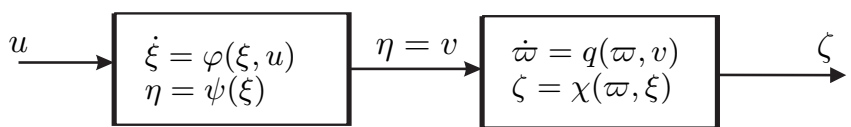

Fig. 5. Interconnection between the nonlinear reduced order model and the generalized signal generator.

Consider the nonlinear system (1) and the nonlinear signal generator (13a). Similar to the arguments for the nonlinear system (1), the moments of the system (2) at $q$ can be characterised by a continuously differentiable function $\chi$ : $\mathbb{R}^{\nu} \times \mathbb{R}^{\nu} \rightarrow \mathbb{R}^{\nu}$ such that $\zeta=\chi(\varpi, \xi)$ satisfies the equation

$$
\left.\left(\frac{\partial \chi(\varpi, \xi)}{\partial \varpi} q(\varpi-\zeta, \psi(\xi))+\frac{\partial \chi(\varpi, \xi)}{\partial \xi} \varphi(\xi, 0)\right)\right|_{\varpi=\gamma(\xi)}=0,
$$

with $\gamma$ such that $\chi(\gamma(\xi), \xi)=0$.

Furthermore, the output $\zeta$ mimics the evolution of $d$ (locally) if there exists a smooth mapping $\alpha$, defined locally around zero, with $\alpha(0)=0$, satisfying $0=$ $\boldsymbol{v}(\rho(\alpha(\xi)), \alpha(\xi))=\chi(\gamma(\xi), \xi)$, such that $\left.\overbrace{d-\zeta}\right|_{x=\alpha(\xi), u=0}=$ 0 , for all $t$. Hence, $\left.\overbrace{d-\zeta}^{i}\right|_{x=\alpha(\xi)}=0$ for all $u$ and $t$ if the moments "match" (locally).

Definition 4: The system (2) matches the moment of (1) at $q$ if there exists a mapping $\alpha$ such that

$$
\begin{aligned}
& \left.\frac{\partial \boldsymbol{v}(\varpi, x)}{\partial x}[f(x, u)-f(x, 0)]\right|_{\varpi=\rho(x), x=\alpha(\xi)} \\
& =\left.\frac{\partial \chi(\varpi, \xi)}{\partial \xi}[\varphi(\xi, u)-\varphi(\xi, 0)]\right|_{\varpi=\gamma(\xi)},
\end{aligned}
$$

where $\rho$ is as in Assumption 4 and $\gamma$ is such that $\chi(\gamma(\xi), \xi)=$ 0 .
In other words, we seek (2) such that the output $\zeta$ of the interconnection with the signal generator (13a) satisfies

$$
\begin{aligned}
\dot{\zeta} & =\left.\frac{\partial \chi(\varpi, \xi)}{\partial \varpi}[q(\varpi, \psi(\xi))-q(\varpi-\zeta, \psi(\xi))]\right|_{\varpi=\gamma(\xi)} \\
& +\left.\frac{\partial \boldsymbol{v}(\varpi, x)}{\partial x}[f(x, u)-f(x, 0)]\right|_{\varpi=\rho(x), x=\alpha(\xi)} .
\end{aligned}
$$

Definition 4 is a generalization of the arguments from [9, Section IV], i.e., a linear system $\dot{\xi}=F \xi+G u, \psi=H \xi$ matches the moments of the linear system $\dot{x}=A x+b u, y=C x$ at $q(\varpi, v)=Q \varpi+R v$ if there exists $\chi(\varpi, \xi)=\varpi+\xi$ such that the signal $\zeta=\chi(\varpi, \xi)$ satisfies equation (21) which becomes $\dot{\zeta}=Q \zeta+\Upsilon B u$.

Remark 1: Following arguments similar to those used in the proof of Lemma 1, $\chi$ satisfies the partial differential equation

$$
\left.\left(\frac{\partial \chi(\varpi, \xi)}{\partial \varpi} q(\varpi-\zeta, \psi(\xi))+\frac{\partial \chi(\varpi, \xi)}{\partial \xi} \varphi(\xi, 0)\right)\right|_{\varpi=\gamma(\xi)}=0 .
$$

For the linear case one may select $\zeta=\varpi+P \xi$. Then $\dot{\zeta}=$ $Q \zeta+\Upsilon B u$ if and only if for $\varpi=\gamma(\xi)=-P \xi$, we have $Q(\varpi-\zeta)+(R H+P F) \xi=0$, i.e., there exists $P$ (invertible) such that $-Q P+R H+P F=0$, yielding the family of reduced order models $\dot{\xi}=(Q-R H) \xi+\Upsilon B u, \psi=H \xi$ as in $[9$, Section IV].

We now compute a family of parametrized models of order $\nu$ that achieve moment matching in the sense of Definition 4.

Theorem 3: Consider the nonlinear system (2) and the signal generator (13a). Suppose Assumption 4 holds and $d$ is as in (13b). Let $\zeta=\chi(\varpi, \xi)$. Assume there exists $\gamma$ such that $\chi(\gamma(\xi), \xi)=0$. Then the system (2) matches the moments of (1) at $q$ (in the sense of Definition 4), if and only if

$$
\begin{aligned}
& \left.\left(\frac{\partial \chi(\varpi, \xi)}{\partial \varpi} q(\varpi-\zeta, \psi(\xi))+\frac{\partial \chi(\varpi, \xi)}{\partial \xi} \varphi(\xi, u)\right)\right|_{\varpi=\gamma(\xi)} \\
& =\left.\frac{\partial \boldsymbol{v}(\varpi, x)}{\partial x}[f(x, u)-f(x, 0)]\right|_{\varpi=\rho(x), x=\alpha(\xi)} .
\end{aligned}
$$

A family of models (2) parametrized in $\psi$ that achieves moment matching is characterised by a mapping $\varphi$ satisfying

$$
\left.\left.\frac{\partial \chi(\varpi, \xi)}{\partial \xi}\right|_{\varpi=\gamma(\xi)} \varphi(\xi, 0)+\frac{\partial \chi(\varpi, \xi)}{\partial \varpi} q(\varpi-\zeta, \psi(\xi))\right]\left.\right|_{\varpi=\gamma(\xi)}=0,
$$

if and only if equation (20) holds.

Proof: Note that

$$
\begin{aligned}
\dot{\zeta}=\frac{\partial \chi(\varpi, \xi)}{\partial \varpi} q(\varpi, \psi(\xi)) & +\frac{\partial \chi(\varpi, \xi)}{\partial \xi}[\varphi(\xi, u)-\varphi(\xi, 0)] \\
& +\frac{\partial \chi(\varpi, \xi)}{\partial \xi} \varphi(\xi, 0) .
\end{aligned}
$$

By Definition (4), moment matching occurs if $\zeta$ satisfies equation (21), which proves the first statement. Since equation 
(22) can be written as

$$
\begin{aligned}
& \left.\left(\frac{\partial \chi(\varpi, \xi)}{\partial \varpi} q(\varpi-\zeta, \psi(\xi))+\frac{\partial \chi(\varpi, \xi)}{\partial \xi} \varphi(\xi, 0)\right)\right|_{\varpi=\gamma(\xi)} \\
& =\left.\frac{\partial \boldsymbol{v}(\varpi, x)}{\partial x}[f(x, u)-f(x, 0)]\right|_{\varpi=\rho(x), x=\alpha(\xi)} \\
& -\left.\frac{\partial \chi(\varpi, \xi)}{\partial \xi}[\varphi(\xi, u)-\varphi(\xi, 0)]\right|_{\varpi=\gamma(\xi)},
\end{aligned}
$$

the second claim also follows directly.

Equations (2) with $\varphi$ satisfying (23) defines a family of nonlinear models of order $\nu$ that match the moments of (1) at $q$. Theorem 3 is a generalised version of the arguments in [9, Section IV], i.e., $\Sigma_{H}: \dot{\xi}=(Q-R H) \xi+\Upsilon B u, \psi=H \xi$, is a family of reduced order models parametrized in $\psi(\xi)=H \xi$ that match the moments $\Upsilon B$ of the linear system $\dot{x}=A x+$ $B u, y=C x$.

Setting $\chi(\varpi, \xi)=\varpi \pm \xi$, the family of reduced order models that achieve moment matching can be described by equations of the form

$$
\begin{aligned}
& \dot{\xi}=-q(\mp \xi, \psi(\xi))+\left.\frac{\partial \boldsymbol{v}(\varpi, x)}{\partial x}[f(x, u)-f(x, 0)]\right|_{\varpi=\mp \xi, x=\alpha(\xi)}, \\
& \eta=\psi(\xi),
\end{aligned}
$$

with $\alpha$ such that $\rho(\alpha(\xi))=\xi$, parametrized in $\psi$.

Moment matching in the sense of Definition 4 is also moment matching by Definition 2, in the sense that the reduced order model (2) that matches the moments of (1) at $q(\varpi, 0)+r v$ in the sense of Definition 4 yields a model that matches the moments of (1) at $\left\{q(\omega, 0), r^{T} \omega\right\}$, with $r=\left.\frac{\partial q(\varpi, v)}{\partial v}\right|_{\square=0}$, in the sense of Definition 2 .

Theorem 4: Consider a nonlinear system (1). Let $q$ be as in (13a). Furthermore, for $\varpi=\omega$, assume that the pair $\left\{q(\omega, 0), r^{T} \omega\right\}$, defines an observable signal generator (3). Suppose that Assumption 4 holds and $d$ is as in (13b). Let $\pi$ be the unique solution of the equation $\frac{\partial \pi(\omega)}{\partial \omega} q(\omega, 0)=$ $f\left(\pi(\omega), r^{T} \omega\right)$. Then a model (2) that matches the moments of (1) at $\left\{q(\omega, 0), r^{T} \omega\right\}$ matches the moment of (1) at $q(\varpi, 0)+r v$ if and only if $\varphi$ satisfies the equation

$$
\frac{\partial \chi(\omega, \xi)}{\partial \xi} \varphi(\xi, 0)=-\frac{\partial \chi(\omega, \xi)}{\partial \omega}[q(\omega-\zeta, 0)-r h(\pi(\xi))]
$$

Proof: We prove the necessity. Assume that (2) matches the moment of (1) at $\left\{q(\omega, 0), r^{T} \omega\right\}$. Then $\psi(\xi)=h(\pi(\xi))$. Let $\zeta=\chi(\omega, \xi)$. Then

$$
\dot{\zeta}=\frac{\partial \chi(\omega, \xi)}{\partial \omega}[q(\omega, 0)+r h(\pi(\xi))]+\frac{\partial \chi(\omega, \xi)}{\partial \xi} \varphi(\xi, u) .
$$

Furthermore,

$$
\begin{aligned}
\dot{\zeta} & =\frac{\partial \chi(\omega, \xi)}{\partial \omega}[q(\omega, 0)+r h(\pi(\xi))] \\
& +\frac{\partial \chi(\omega, \xi)}{\partial \xi}[\varphi(\xi, u)-\varphi(\xi, 0)]+\frac{\partial \chi(\omega, \xi)}{\partial \xi} \varphi(\xi, 0) .
\end{aligned}
$$

If (2) matches the moment at $q(\omega, 0)+r v$ in the sense of Definition 4 then (21) holds and the claim follows.

The sufficiency uses similar arguments and follows after employing equation (21), hence the proof is omitted.

\section{B. Moment matching with asymptotic stability}

Since, in practice, asymptotically stable reduced order models are desirable, in this section, we briefly discuss the problem of achieving model reduction by moment matching such that the approximant has an asymptotically stable equilibrium. To this end, note that the linearisation of the model $(2)$ at $(0,0)$, that matches the moments $\Upsilon B$ of the linearisation of system (1), is a model of the form $\Sigma_{H}: \dot{\xi}=(Q-R H) \xi+\Upsilon B u, \psi=$ $H \xi$, with $Q=\left.\frac{\partial q(\varpi, v)}{\partial \varpi}\right|_{(\varpi, v)=(0,0)}, R=\left.\frac{\partial q(\varpi, v)}{\partial v}\right|_{(\varpi, v)=(0,0)}$, $H=\left.\frac{\partial \psi(\xi)}{\partial \xi}\right|_{\xi=0}$. Assuming that the pair $(Q, R)$ is controllable, there exists $H$ such that the eigenvalues of $Q-R H$ are in the open left half plane, hence, there exists a model (2) that has an asymptotically stable equilibrium at $(0,0)$, see, e.g., [22], [27] for details. Note that this is not a necessary condition.

\section{Moment matching at $\{s, l\}$ and at $q$}

In this section we compute the reduced order model (models) that matches (match) moments at $\{s, l\}$ and at $q$, simultaneously. This is done by a selection of the free parameters, i.e., from the classes of models that achieve moment matching we identify the one matching two sets of moments, simultaneously. This is the nonlinear extension of the problem of finding linear reduced order models that match a number of moments equal to double their dimension, see, e.g., [1, Chapter 11] and the references therein.

Consider the simultaneous interconnection of system (1) with the signal generator (3) defined by the relation $u=\theta$ and with the signal generator (13) defined by the relation $y=v$. We call this a two-sided interconnection of system (1) with the signal generators (3) and (13), see Figure 6.

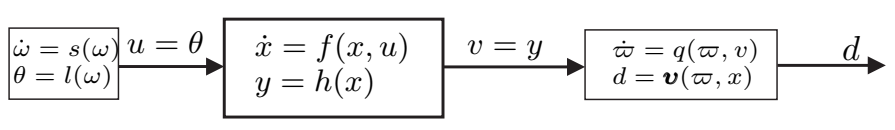

Fig. 6. Diagram illustrating the two-sided interconnection of (1) with the signal generators (3) and (13).

In this case the moments of system (1) at $\{s, l\}$ and at $q$ are characterised simultaneously by the evolution of the signal $d$ if and only if the solution $\boldsymbol{v}$ of (15) satisfies a certain equation on the manifold $x=\pi(\omega)$, restricted to $\varpi=\rho(x)$, with $\rho$ such that Assumption 4 holds.

Proposition 1: Consider the interconnection between the system (1) and the signal generators (3) and (13) defined by $\theta=u$ and $v=y$, respectively. Then the moments of (1) at $\{s, l\}$ and at $q$ are characterised, simultaneously, by the signal $d$ satisfying equation (18) if and only if $\boldsymbol{v}$ satisfies

$$
\begin{aligned}
& \left.\left(\frac{\partial \boldsymbol{v}(\varpi, x)}{\partial \varpi} q(\varpi-d, h(\pi(\omega)))+\frac{\partial \boldsymbol{v}(\varpi, x)}{\partial x} f(x, 0)\right)\right|_{\varpi=\rho(x), x=\pi(\omega)} \\
& =0,
\end{aligned}
$$

where $\rho$ and $\boldsymbol{v}$ are such that Assumption 4 holds, and $\pi$ is the unique solution of (5).

Proof: Let $\mathcal{M}=\{(\omega, x) \mid x=\pi(\omega)\}$, where $\pi$ is the unique solution of (5). Then $d=\boldsymbol{v}(\varpi, \pi(\omega))$ on $\mathcal{M}$. Hence, 


$$
\text { for } \begin{aligned}
\varpi & =\rho(x), \\
\dot{d} & =\left.\frac{\partial \boldsymbol{v}(\varpi, x)}{\partial \varpi}\right|_{x=\pi(\omega)} \dot{\varpi}+\left.\frac{\partial \boldsymbol{v}(\varpi, x)}{\partial x}\right|_{x=\pi(\omega)} \frac{\partial \pi(\omega)}{\partial \omega} \dot{\omega} \\
& =\left.\frac{\partial \boldsymbol{v}(\varpi, x)}{\partial \varpi}\right|_{x=\pi(\omega)} q(\varpi, h(\pi(\omega))) \\
& +\left.\frac{\partial \boldsymbol{v}(\varpi, x)}{\partial x}\right|_{x=\pi(\omega)} \frac{\partial \pi(\omega)}{\partial \omega} s(\omega),
\end{aligned}
$$

where $s$ is as in (3). By (5)

$$
\begin{aligned}
\dot{d} & =\left.\frac{\partial \boldsymbol{v}(\varpi, x)}{\partial \varpi}\right|_{x=\pi(\omega)} q(\varpi, h(\pi(\omega))) \\
& +\left.\frac{\partial \boldsymbol{v}(\varpi, x)}{\partial x}\right|_{x=\pi(\omega)} f(\pi(\omega), l(\omega)),
\end{aligned}
$$

where $l$ is as in (3). Restricting the behaviour of $d$ to the manifold $\varpi=\rho(x)$ yields the claim, by Lemma 1 .

The next result provides the conditions that the reduced order model (2) must satisfy to match the moments of (1) at $\{s, l\}$ and at $q$, simultaneously.

Theorem 5: Consider the interconnection between the system (2) and the signal generators (3) and (13) defined by $\theta=u$ and $w=\eta$, respectively. Let $\boldsymbol{v}$ be such that Assumption 4 holds. Let $\gamma$ be such that $\chi(\gamma(\xi), \xi)=0$, with $\chi$ as in Definition 4 and let $\pi$ be the unique solution of (5). Then the following statements are equivalent.

1) The models (2) match the moments of (1) at $\{s, l\}$ and at $q$, simultaneously.

2) There exists a coordinate transformation $\xi=p(\omega)$, with $p$ satisfying (6), such that $\varphi$ and $\psi$ satisfy the equations

$$
\begin{aligned}
& \left.\frac{\partial \chi(\varpi, \xi)}{\partial \xi}\right|_{\varpi=\gamma(\xi), \xi=p(\omega)} \varphi(p(\omega), l(\omega)) \\
& =\left.\frac{\partial \boldsymbol{v}(\varpi, x)}{\partial x}\right|_{\varpi=\gamma(p(\omega)), x=\pi(\omega)} \frac{\pi(\omega)}{\partial \omega} s(\omega) \\
& -\left.\frac{\partial \chi(\varpi, \xi)}{\partial \varpi} q(\varpi-\zeta, h(\pi(\xi)))\right|_{\varpi=\gamma(\xi), \xi=p(\omega)} \\
& -\left.\frac{\partial \boldsymbol{v}(\varpi, x)}{\partial x} f(x, 0)\right|_{\varpi=\gamma(p(\omega)), x=\pi(\omega)}
\end{aligned}
$$

and

$$
\psi(p(\omega))=h(\pi(p(\omega))) .
$$

Proof: We first prove that "1) $\Rightarrow 2$ )". Consider the interconnection between system (2) and the signal generators (3) and (13) defined by $\theta=u$ and $w=\eta$, respectively. Note that if (2) matches the moments of (1) at $\{s, l\}$, then there exists a coordinate transformation $\xi=p(\omega)$ such that $\psi(p(\omega))=$ $h(\pi(p(\omega)))$, where $p$ satisfies $\varphi(p(\omega), l(\omega))=\frac{\partial p(\omega)}{\partial \omega} s(\omega)$. Let $\zeta=\chi(\varpi, p(\omega))$. Let $\gamma$ be such that $\chi(\gamma(\xi), \xi)=0$. Then $\dot{\zeta}=\frac{\partial \chi(\varpi, p(\omega))}{\partial \varpi} q(\varpi, \psi(p(\omega)))+\left.\frac{\partial \chi(\varpi, \xi)}{\partial \xi}\right|_{\xi=p(\omega)} \varphi(p(\omega), l(\omega))$. If, in addition, (2) matches the moment at $q(\varpi, v)$, then $\dot{\zeta}$ satisfies (21) and by Theorem 4, the claim follows.

We now prove " 2$) \Rightarrow 1)$ ". Note that if there exists $p$ such that $\psi(p(\omega))=h(\pi(p(\omega)))$ and $\phi(p(\omega))=h(\pi(p(\omega)))$, where $p$ satisfies (6), then (2) matches the moment at $\{s, l\}$, by Definition 2. Furthermore, (27) can be written as

$$
\begin{aligned}
& \left.\frac{\partial \chi(\varpi, \xi)}{\partial \xi}\right|_{\varpi=\gamma(\xi), \xi=p(\omega)} \varphi(p(\omega), l(\omega)) \\
& =\left.\frac{\partial \boldsymbol{v}(\varpi, x)}{\partial x}\right|_{\varpi=\gamma(p(\omega)), x=\pi(\omega)} \frac{\pi(\omega)}{\partial \omega} s(\omega) \\
& -\left.\frac{\partial \chi(\varpi, \xi)}{\partial \varpi} q(\varpi-\zeta, h(\pi(\xi)))\right|_{\varpi=\gamma(\xi), \xi=p(\omega)} \\
& -\left.\frac{\partial \boldsymbol{v}(\varpi, x)}{\partial x} f(x, 0)\right|_{\varpi=\gamma(p(\omega)), x=\pi(\omega)} \\
& \pm\left.\frac{\partial \boldsymbol{v}(\varpi, x)}{\partial x} f(x, l(\omega))\right|_{\varpi=\gamma(p(\omega)), x=\pi(\omega)} .
\end{aligned}
$$

By assumption, $\pi$ is the unique solution of the equation $\frac{\partial \pi(\omega)}{\partial \omega}=f(\pi(\omega), l(\omega))$. Hence (28) becomes (22) and, by Theorem 4, the claim follows.

Remark 2: In the linear case, by [21, Proposition 1], a reduced order model $\dot{\xi}=F \xi+G u, \psi=H \xi$, that matches the moments at $\sigma(S)$ and at $\sigma(Q)$, simultaneously, satisfies (27) which becomes $Q P-P S=\Upsilon B L-R C \Pi$, for $P=\Upsilon \Pi$, provided the matrix $\Upsilon \Pi$ is invertible, with $\Pi$ and $\Upsilon$, the unique solutions of the Sylvester equations $A \Pi+B L=\Pi S$ and $Q \Upsilon=\Upsilon A+R C$, respectively.

A more explicit condition is achieved for the family of models (24), i.e., in the family of models (24) that match the moment at $q$, there exist parameters $\psi$ that identify the model(s) that also match the moments of (1) at $\{s, l\}$.

Corollary 1: Consider a model that matches the moments of (1) at $q$, described by equations (24). Then the model (24) also matches the moments of (1) at $\{s, l\}$ if and only if there exists a coordinate transformation $\xi=p(\omega)$ such that $\psi$ satisfies the equation

$$
\begin{aligned}
& \left.\frac{\partial \boldsymbol{v}(\varpi, x)}{\partial x}\right|_{\varpi=\mp p(\omega), x=\pi(\omega)} \frac{\pi(\omega)}{\partial \omega} s(\omega) \\
& +\left.q(\mp p(\omega), \psi(p(\omega)))\right|_{\psi(p(\omega))=h(\pi(p(\omega)))} \\
& =\left.\frac{\partial \boldsymbol{v}(\varpi, x)}{\partial x}[f(x, u)-f(x, 0)]\right|_{x=\pi(\omega), \varpi=\mp p(\omega)},
\end{aligned}
$$

where $v$ and $\rho$ are such that Assumption 4 holds, with $\pi$ the unique solution of (5).

Proof: By (24), for $\xi=p(\omega)$, we have

$$
\begin{aligned}
\varphi(p(\omega), l(\omega)) & =-q(\mp p(\omega), \psi(p(\omega))) \\
& +\left.\frac{\partial \boldsymbol{v}(\varpi, x)}{\partial x}[f(x, u)-f(x, 0)]\right|_{x=\pi(\omega), \varpi=\mp p(\omega)} .
\end{aligned}
$$

Substituting this relation in (27) and noting that by (24), $\chi(\varpi, \xi)=\varpi \pm \xi$, yields the claim.

Note that, unlike the linear case, equation (29) may not have a unique solution, hence there might be one or more models (24) that achieve moment matching of (1) at $\{s, l\}$ and at $q$, simultaneously.

\section{Nonlinear Petrov-Galerkin projection-based moment matching at $\{s, l\}$ and at $q$}

In this section we compute the subfamily of nonlinear Petrov-Galerkin projection-based models (11) from Theorem 
2 that achieve moment matching at $\{s, l\}$ and at $q$, simultaneously.

Proposition 2: Consider the family of models (11). Suppose that the assumptions from the preamble of Theorem 2 hold. Then (11) matches the moments of (1) at $\{s, l\}$ and at $q$ simultaneously if there exists a coordinate transformation $\xi=p(\omega)$ such that $\pi \circ p$ and $\varrho$ in (10) satisfy the equation

$$
\begin{aligned}
& \left.\left(\frac{\partial \varrho(x)}{\partial x}-\frac{\partial \chi(\varpi, \xi)}{\partial \xi}\right)\right|_{\varpi=\gamma(p(\omega)), x=\pi(\omega)} f(\pi(p(\omega)), l(\omega)) \\
& =\left.\frac{\partial \chi(\varpi, \xi)}{\partial \varpi} q(\varpi, h(\pi(\xi)))\right|_{\varpi=\gamma(p(\omega)), \xi=p(\omega)} \\
& +\left.\frac{\partial \boldsymbol{v}(\varpi, x)}{\partial x} f(x, 0)\right|_{\varpi=\gamma(p(\omega)), x=\pi(p(\omega))},
\end{aligned}
$$

with $\boldsymbol{v}$ such that Assumption 4 holds and $\gamma$ such that $\left.\chi(\gamma(\xi), \xi)\right|_{\xi=p(\omega)}=0$.

Proof: The result is a direct application of Theorem 5 to the family of models (11). In fact, substituting $\varphi(\pi(p(\omega)), l(\omega))=\left.\frac{\partial \varrho(x)}{\partial x} f(x, l(\omega))\right|_{x=\pi(\xi), \xi=p(\omega)}$, as in (11), into (27) and utilising equation (10) yields the claim.

\section{THE NONLINEAR INPUT-AFFINE CASE}

In this section we apply the results of Section III to the case of nonlinear systems affine in the input, often arising in applications. In this case, the aforementioned results yield explicit families of models that achieve moment matching.

\section{A. Moment matching at $q(\varpi, v)=\bar{q}(\varpi)+r(\varpi) v$}

Consider a nonlinear system (1) with $f(x, u)=\bar{f}(x)+$ $g(x) u$, where $\bar{f}$ and $g$ are smooth mappings, with $\bar{f}(0)=$ 0 and $h(0)=0$. Furthermore, consider a generalized signal generator (13), with $q(\varpi, v)=\bar{q}(\varpi)+r(\varpi) v$, where $\bar{q}$ and $r$ are smooth mappings, such that $\bar{q}(0)=0$. Let $d=\varpi \pm \overline{\boldsymbol{v}}(x)$, with $\overline{\boldsymbol{v}}$ a smooth mapping such that $\overline{\boldsymbol{v}}(0)=0$. By Lemma 1, $d$ satisfies equation (18) if and only if $\bar{v}$ is the solution of the nonlinear Sylvester-like equation

$$
\bar{q}(\mp \overline{\boldsymbol{v}}(x))+r(\mp \overline{\boldsymbol{v}}(x)) h(x)+\frac{\partial \overline{\boldsymbol{v}}(x)}{\partial x} \bar{f}(x)=0 .
$$

Note that $\rho(x)=\mp \overline{\boldsymbol{v}}(x)$ with $\rho$ such that Assumption 4 holds.

By Definition 3, the moments of (1) at $\{\bar{q}, r\}$ are described by $\pm \frac{\partial \overline{\boldsymbol{v}}(x)}{\partial x} g(x)$.

Let $\zeta=\chi(\varpi, \xi)=\varpi \pm \xi$. In this case, $\gamma(\xi)=-\xi$ yielding $\zeta=0$. By construction, $\xi=\overline{\boldsymbol{v}}(x)$. Imposing the matching condition as in Theorem 3, the equations

$\Sigma_{\psi(\xi)}:\left\{\begin{array}{l}\dot{\xi}=-\bar{q}(\mp \xi)-r(\mp \xi) \psi(\xi)+\left.\frac{\partial \overline{\boldsymbol{v}}(x)}{\partial x} g(x)\right|_{x=\alpha(\xi)} u, \\ \eta=\psi(\xi),\end{array}\right.$

where $\alpha$ is such that $\overline{\boldsymbol{v}}(\alpha(\xi))=\mp \xi$, with $\xi(t) \in \mathbb{R}^{\nu}$, define a class of models of order $\nu$, parametrized in $\psi$, that match the moments of (1) at $\{\bar{q}, r\}$.

Moment matching in the sense of Definition 4 is related to moment matching in the sense of Definition 2, i.e., a model
$\Sigma_{\psi(\xi)}$ matches the moments of (1) at $\{\bar{q}, r\}$ in the sense of Definition 4 if and only if $\Sigma_{\psi(\xi)}$ matches the moments of (1) at $\{\bar{q}, l\}$, with $l(\varpi)=r^{T}(0) \varpi$, in the sense of Definition 2, for $\omega=\varpi$. For the sake of clarity, we only consider the case $\zeta=\varpi+\xi$.

Theorem 6: Consider a nonlinear observable system described by the equations (1). Let $\bar{q}(\omega)+r(0) v$ define the generalized signal generator in (13). Furthermore, assume that the pair $\{-\bar{q}, l\}$, with $l(\omega)=r^{T}(0) \omega$, defines an observable signal generator (3). Consider the systems $\Sigma_{\psi(\xi)}$ in (32) and $\Sigma_{\delta(\xi)}$ in (8). Suppose that Assumptions 1 and 4 hold. The following statements hold.

1) $\Sigma_{\psi(\xi)}$, described by equations (32), that matches the moments of (1) at $\{\bar{q}, r\}$ in the sense of Definition 4, matches the moment at $\left\{-\bar{q}(-\omega), r^{T}(0) \omega\right\}$ if and only if $r(0) h(\pi(\omega))=\left.\frac{\partial \overline{\boldsymbol{v}}(x)}{\partial x} g(x)\right|_{x=\alpha(\omega)} r^{T}(\omega) r^{T}(0) \omega$ and $\psi(\omega)=h(\pi(\omega))$.

2) $\Sigma_{\delta(\xi)}$, described by equations (8), that matches the moments of (1) at $\left\{-\bar{q}(-\omega), r^{T}(0) \omega\right\}$, in the sense of Definition 2, matches the moment of at $\{\bar{q}, r\}$ if and only if $r(0) h(\pi(\xi))=\delta(\xi) r^{T}(0) \xi$ and $\delta(\xi)=$ $\left.\frac{\partial \overline{\boldsymbol{v}}(x)}{\partial x} g(x)\right|_{x=\alpha(\xi)}$.

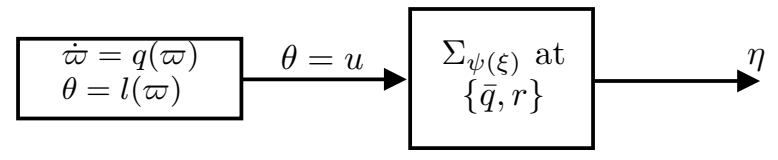

Fig. 7. Diagram illustrating statement 1) of Theorem 6.

Proof: We prove statement 1). Let $\Sigma_{\psi(\xi)}$ be as in (32). Then $\Sigma_{\psi(\xi)}$ matches the moment at $\left\{-\bar{q}(-\omega), r^{T}(0) \omega\right\}$ if and only if, by Definition 2, there exists $p$ satisfying $\psi(p(\omega))=h(\pi(p(\omega)))$, such that the equation $-\bar{q}(-p(\omega))+$ $r \psi(p(\omega))+\left.\frac{\partial \overline{\boldsymbol{v}}(x)}{\partial x} g(x)\right|_{\xi=\overline{\boldsymbol{v}}(x), \xi=p(\omega)} r^{T}(0) \omega=-\frac{\partial p(\omega)}{\partial \omega} \bar{q}(-\omega)$ has the unique solution $p(\omega)=\omega$. This holds if and only if $r(0) h(\pi(\omega))=\left.\frac{\partial \overline{\boldsymbol{v}}(x)}{\partial x} g(x)\right|_{x=\alpha(\omega)} r^{T}(0) \omega$.

We now prove statement 2 ). Consider a system $\Sigma_{\delta(\xi)}$ that matches the moment of (1) at $\left\{-\bar{q}(-\omega), r^{T}(0) \omega\right\}$. Then, $\Sigma_{\delta(\xi)}$ matches the moment at $\bar{q}(\omega)+r(0) v$ if and only if $\zeta=\omega+\xi$ satisfies (21). Note that

$\dot{\zeta}=\dot{\omega}+\dot{\xi}=\bar{q}(\omega)+r(0) h(\pi(\xi))-\bar{q}(-\xi)-\delta(\xi) r^{T}(0) \xi+\delta(\xi) u$.

Then, by (21) and Definition $4, \zeta$ must satisfy

$$
\dot{\zeta}=\bar{q}(\omega)-\bar{q}(\underbrace{\omega-\zeta}_{-\xi})+\left.\frac{\partial \overline{\boldsymbol{v}}(x)}{\partial x} g(x)\right|_{x=\alpha(\xi)} u,
$$

for all $\xi$ and $u$. This holds if and only if $\delta(\xi)=$ $\left.\frac{\partial \overline{\boldsymbol{v}}(x)}{\partial x} g(x)\right|_{x=\alpha(\xi)}$ and $r(0) h(\pi(\xi))=\delta(\xi) r^{T}(0) \xi$.

\section{B. Moment matching at $\{s, l\}$ and at $\{\bar{q}, r\}$}

In this section we give the nonlinear counterpart of [21, Proposition 1], i.e., we compute the subfamily of models of order $\nu$ that match the moments at $\{s, l\}$ and the moments at $\{\bar{q}, r\}$, of a given nonlinear system, simultaneously. 
Proposition 3: Consider the nonlinear system (1) and the signal generators (3) and (13a). Let $\pi$ be the unique solution of (5) and $\bar{v}$ be the unique solution of (31). Let $\alpha$ be such that $\overline{\boldsymbol{v}}(\alpha(\xi))=\mp \xi$. Then the following statements hold.

1) There exist a subfamily of models $\Sigma_{\delta(\xi)}$ as in (8) that match the moments of (1) at $\{s, l\}$ and $\{\bar{q}, r\}$ simultaneously if and only if $\delta$ satisfies the equation

$$
\frac{\partial p(\xi)}{\partial \xi} \delta(\xi)=\left.\frac{\partial \overline{\boldsymbol{v}}(x)}{\partial x} g(x)\right|_{x=\alpha(\xi)} l(\xi),
$$

where $p: \mathbb{R}^{\nu} \rightarrow \mathbb{R}^{\nu}$ satisfies the equation

$$
\begin{aligned}
\bar{q}( \pm p(\xi))+\frac{\partial p(\xi)}{\partial \xi} s(\xi) & =\left.\frac{\partial \overline{\boldsymbol{v}}(x)}{\partial x} g(x)\right|_{x=\alpha(\xi)} l(\xi) \\
& \mp r h(\pi(\xi)) .
\end{aligned}
$$

2) There exist a subfamily of models $\Sigma_{\psi(\xi)}$ as in (32) that match the moments of (1) at $\{s, l\}$ and $\{\bar{q}, r\}$ simultaneously if and only if

$$
\psi(p(\omega))=h(\pi(\omega)),
$$

where $p: \mathbb{R}^{\nu} \rightarrow \mathbb{R}^{\nu}$ satisfies the equation

$$
\begin{aligned}
\bar{q}( \pm p(\omega))+\frac{\partial p(\omega)}{\partial \omega} s(\omega) & =\left.\frac{\partial \boldsymbol{v}(x)}{\partial x} g(x)\right|_{x=\alpha(\xi), \xi=p(\omega)} l \\
& \mp r h(\pi(\omega)) .
\end{aligned}
$$

Proof: It follows arguments identical to the proof of Theorem 6, hence it is omitted.

Remark 3: Assuming that $p$ uniquely satisfies equation (34), there exists a unique model $\Sigma_{\delta(\xi)}$ that matches the moments of (1) at $\{s, l\}$ and at $\{\bar{q}, r\}$, simultaneously. Furthermore, if $p(\xi)$

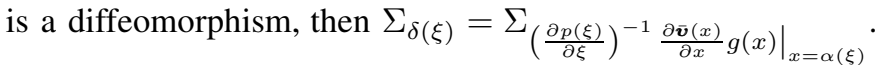
Similarly, assuming that $p$ uniquely satisfies equation (36), there exists a unique model $\Sigma_{\psi(\xi)}$ that matches the moments of (1) at $\{s, l\}$ and $\{\bar{q}, r\}$. Finally, if we assume that $p$ is the identity, then statement 1) and statement 2) from Proposition 3 are equivalent and moreover

$$
\Sigma_{\delta(\xi)}=\Sigma_{\left.\frac{\partial \overline{\boldsymbol{v}}(x)}{\partial x} g(x)\right|_{x=\alpha(\xi)}}=\Sigma_{h(\pi(\xi))}=\Sigma_{\psi(\xi)}
$$

is the unique model of order $\nu$ that matches the moments of (1) at $\{s, l\}$ and at $\{\bar{q}, r\}$, simultaneously.

\section{ILLUSTRATIVE EXAMPLES}

\section{A. An academic example}

Consider the one-dimensional system described by the equations

$$
\begin{aligned}
& \dot{x}=-x-x^{3}+u, \\
& y=x,
\end{aligned}
$$

and let the signal generator (3) be $\dot{\omega}=0, \theta=\omega$. Consider the equation $\pi^{3}(\omega)+\pi(\omega)-\omega=0$, which is of the form (5).
This equation has the unique solution

$$
\begin{aligned}
\pi(\omega) & =\frac{1}{6} \sqrt[3]{108 \omega+12 \sqrt{12+81 \omega^{2}}} \\
& -2 \frac{1}{\sqrt[3]{108 \omega+12 \sqrt{12+81 \omega^{2}}}}, \pi(0)=0 .
\end{aligned}
$$

A family of first order models, parametrized in $\delta$, that match the moment of (37) at $\{0, \omega\}$ is

$$
\begin{aligned}
\dot{\xi} & =\delta(\xi)(u-\xi), \\
\eta & =\frac{1}{6} \sqrt[3]{108 \xi+12 \sqrt{12+81 \xi^{2}}} \\
& -2 \frac{1}{\sqrt[3]{108 \xi+12 \sqrt{12+81 \xi^{2}}}}
\end{aligned}
$$

for any $\delta$ that satisfies (9).

Consider now the generalized signal generator $\dot{\varpi}=r v, r \in$ $\mathbb{R}, d=\varpi+\boldsymbol{v}(x)$. Equation (15) becomes $\frac{\partial \boldsymbol{v}(x)}{\partial x}\left(x^{3}+x\right)=r x$. The (unique) solution of this differential equation such that $\boldsymbol{v}(0)=0$, is $\boldsymbol{v}(x)=r \arctan x$. Let $\xi=\boldsymbol{v}(x)$. Note that, since the arctan function is invertible, there exists (locally) $\alpha$ that satisfies $\alpha(\boldsymbol{v}(x))=x$, given by $\alpha(\xi)=\tan \frac{\xi}{r}$. Hence the moment of (37) at $q(\varpi, v)=r v$, as in Definition 3, is $\left.\frac{\partial \boldsymbol{v}(x)}{\partial x} g(x)\right|_{x=\alpha(\xi)}=\left.\frac{r}{1+x^{2}}\right|_{x=\alpha(\xi)}$. Imposing the moment matching conditions then $\left.\frac{\partial \boldsymbol{v}(x)}{\partial x} g(x)\right|_{x=\alpha(\xi)}=$ $\left.\frac{\partial \boldsymbol{v}(x)}{\partial x} g(x)\right|_{x=\alpha(\xi)}=\frac{r}{1+\tan ^{2} \frac{\xi}{r}}=r \cos ^{2}\left(\frac{\xi}{r}\right)$. A family of first order models parametrized by $\psi$ that match the moment of (37) at $r v$ is given by the equations

$$
\begin{aligned}
\dot{\xi} & =r\left[\cos ^{2}\left(\frac{\xi}{r}\right) u-\psi(\xi)\right], \\
\eta & =\psi(\xi) .
\end{aligned}
$$

Let $r=1$. Consider equation (36), i.e.,

$$
\pi(\omega(\xi))-\xi \cos ^{2}(\xi)=0,
$$

with $\pi$ as in (38). By Proposition 3, there exists a model (40) with $\psi(\xi)=\pi(\omega(\xi))=\xi \cos ^{2} \xi$ that matches both the moment as in (38) and the moment of (37) at rv, i.e.,

$$
\begin{aligned}
\dot{\xi} & =\cos ^{2} \xi(u-\xi), \\
\eta & =\xi \cos ^{2} \xi .
\end{aligned}
$$

\section{B. Reduced order model of a DC-to-DC Cuk converter}

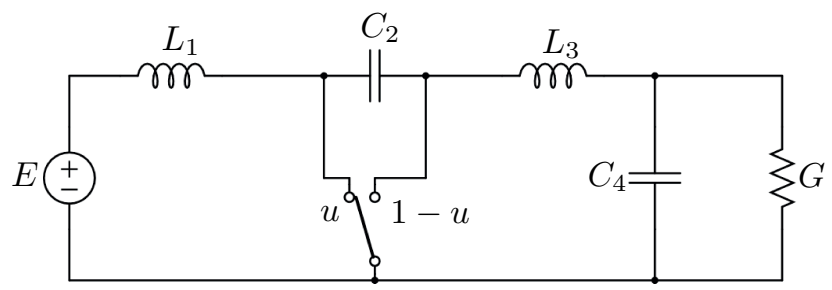

Fig. 8. DC-to-DC Ćuk converter circuit. 
The averaged model of a DC-to-DC Ćuk converter is given by the equations, see [28],

$$
\begin{aligned}
L_{1} \frac{d i_{1}(t)}{d t} & =-(1-u) v_{2}+E, \\
C_{2} \frac{d v_{2}(t)}{d t} & =(1-u) i_{1}+u i_{3}, \\
L_{3} \frac{d i_{3}(t)}{d t} & =-u v_{2}-v_{4}, \\
C_{4} \frac{d v_{4}(t)}{d t} & =i_{3}-G v_{4}, \\
y & =v_{4},
\end{aligned}
$$

where $i_{1}(t) \in \mathbb{R}^{+}$and $i_{3}(t) \in \mathbb{R}^{-}$describe currents, $v_{2}(t) \in \mathbb{R}^{+}$and $v_{4}(t) \in \mathbb{R}^{-}$describe voltages, $L_{1}, C_{2}, L_{3}, C_{4}$ and $G$ are positive parameters, $E \in \mathbb{R}$ and $u(t) \in(0,1)$ is a continuous control signal which represents the slew rate of a Pulse-Width-Modulation circuit used to control the switch position in the converter. Let the first equation of the signal generator (13a) be described by $q(\varpi, v)=\varpi+v$. Let $x^{T}=$ $\left[\begin{array}{llll}x_{1} & x_{2} & x_{3} & x_{4}\end{array}\right]^{T}=\left[\begin{array}{llll}i_{1} & v_{2} & i_{3} & v_{4}\end{array}\right]^{T} \in \mathbb{R}^{4}$ be the state of the model. Let the output (13b) be $d=\varpi+\boldsymbol{v}(x)$. Because of the bilinear form of the model, equation (15) becomes $\frac{\partial \boldsymbol{v}(x)}{\partial x} f(x, 0)+$ $C_{4} x_{4}=0$, with $f(x, u)=\left[\left(-(1-u) x_{2}+E\right) / L_{1}((1-\right.$ $\left.\left.u) x_{1}+u x_{3}\right) / C_{2}\left(-u x_{2}-x 4\right) / L_{3}\left(x_{3}-G x_{4}\right) / C_{4}\right]$ and has the solution $\boldsymbol{v}(x)=\frac{L_{3}}{C_{4} L_{3}+G L_{3}-1} x_{3}+\frac{C_{4} L_{3}}{C_{4} L_{3}+G L_{3}-1} x_{4}+$ higher order terms. Taking the linear part of the solution, a family of first order models of the form (24) that match the moment of (43) at $C_{4} v$ is described by the equations

$$
\begin{aligned}
\dot{\xi} & =-C_{4} \psi(\xi)-\frac{v_{2}}{C_{4} L_{3}+G L_{3}-1} u, \\
\eta & =\psi(\xi),
\end{aligned}
$$

parametrized in $\psi$ and $v_{2}$. Note that $v_{2}$ is a function of $\xi$ such that Assumption 4 holds. Now, select $\{s(\omega), l(\omega)\}=\{0, \omega\}$. Then equation (5) has the unique solution

$$
\pi(\omega)=\left[\begin{array}{c}
G E \frac{\omega^{2}}{(1-\omega)^{2}} \\
E \frac{1}{1-\omega} \\
G E \frac{\omega}{\omega-1} \\
E \frac{\omega}{\omega-1}
\end{array}\right] .
$$

Hence $h(\pi(p(\omega)))=\frac{p(\omega)}{p(\omega)-1}$ and equation (29) has the unique solution $p(\omega)=\omega$, for $v_{2}$ selected as $v_{2}(\omega)=\omega\left(C_{4} L_{3}+\right.$ $\left.G L_{3}-1\right) /(\omega-1)$. By Corollary 1, the unique first order model, from the family of models (44), that matches the moment at $\varpi+v$ and the moment at $\{0, \omega\}$, simultaneously, is given by the equations

$$
\begin{aligned}
\dot{\omega} & =-\omega-\frac{\omega}{\omega-1}(u+1), \\
\eta & =\frac{\omega}{\omega-1} .
\end{aligned}
$$

Figure 9(a) shows the outputs of the systems (43) and (45), respectively, for $u(t)=\frac{1(t)}{2}$, where $1(t)$ denotes the Heaviside function. Figure 9(b) shows the comparison of the outputs of the systems (43) and (45), respectively, for $u(t)=\epsilon e^{t}$, with $\epsilon>0$. This input has been chosen since we are matching at $\dot{\varpi}=\varpi+v$. Note that $u(t)<1$ for large values of $t>0$ if $\epsilon$ is in the neighbourhood of zero.

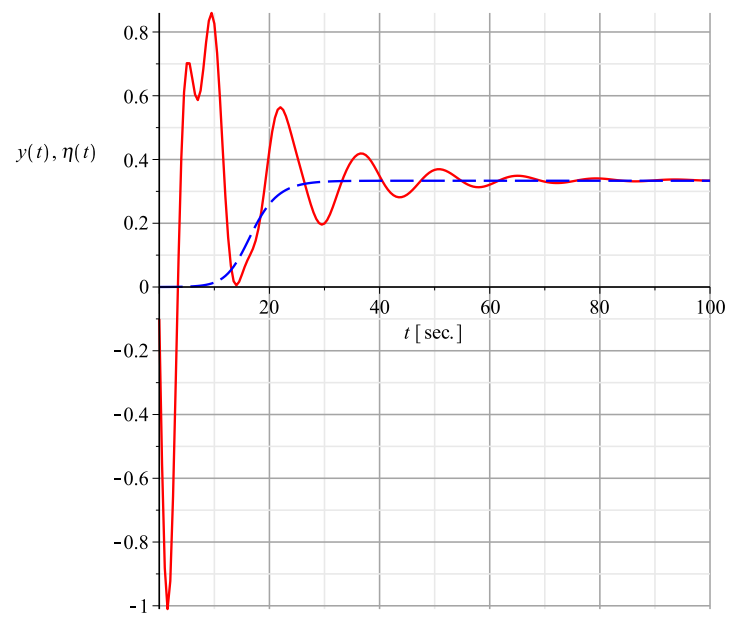

(a)

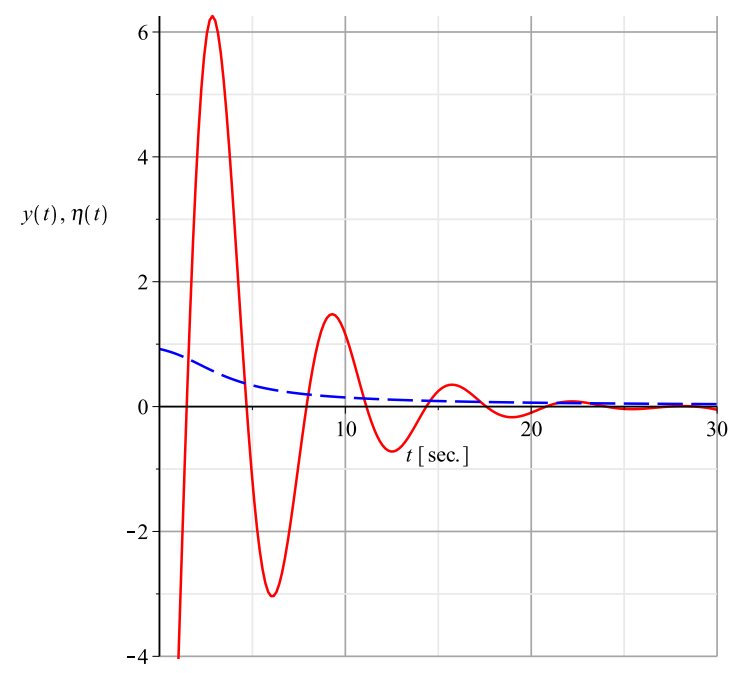

(b)

Fig. 9. Response of the Ćuk DC-to-DC converter (red, solid line) and of the first order model (45) (blue, dashed line) for input $u=0.5 \cdot 1(t)$ (a) and $u(t)=10^{-15} \cdot e^{t}$, with $\omega(0)=-12(\mathrm{~b})$.

\section{Conclusions}

In this paper we have extended the results from [9], [14]. In particular we have presented a time-domain notion of moment for general nonlinear systems in terms of the evolution of the output of a generalized signal generator driven by the nonlinear system. In addition, we have defined a new notion of moment matching and presented the class of (nonlinear) parametrized reduced order models that achieve moment matching. Furthermore, we have established relations 
with existing notions of moment, showing that the families of reduced order models that achieve nonlinear moment matching are equivalent. Furthermore, we have computed the reduced order model that matches moments at two sets of interpolation points, simultaneously, i.e., the number of interpolation points is twice the order of the model.

\section{REFERENCES}

[1] A. C. Antoulas, Approximation of large-scale dynamical systems. Philadelphia: SIAM, 2005.

[2] W. B. Gragg and A. Lindquist, "On the partial realization problem," Linear Algebra \& its Applications, vol. 50, pp. 277-319, 1983.

[3] P. van Dooren, "The Lanczos algorithm and Padé approximation," Benelux Meeting on Systems and Control, 1995, Minicourse.

[4] K. Gallivan and P. V. Dooren, "Rational approximations of pre-filtered transfer functions via the Lanczos algorithm," Numerical Algorithms, vol. 20, pp. 331-342, 1999.

[5] C. de Villemagne and R. E. Skelton, "Model reductions using a projection formulation," Int. J. Control, vol. 46, pp. 2141-2169, 1987.

[6] A. C. Antoulas, J. A. Ball, J. Kang, and J. C. Willems, "On the solution of the minimal rational interpolation problem," Linear Algebra \& its Applications, vol. 137/138, pp. 511-573, 1990.

[7] I. M. Jaimoukha and E. M. Kasenally, "Implicitly restarted Krylov subspace methods for stable partial realizations," SIAM J. Matrix Anal. Appl., vol. 18, pp. 633-652, 1997.

[8] A. Astolfi, "Model reduction by moment matching for linear and nonlinear systems," IEEE Trans. Autom. Contr., vol. 50, no. 10, pp. 2321-2336, 2010

[9] — - "Model reduction by moment matching, steady-state response and projections," in Proc. 49th IEEE Conf. on Decision and Control, 2010, pp. $5344-5349$.

[10] K. Gallivan, A. Vandendorpe, and P. V. Dooren, "Sylvester equations and projection based model reduction," J. Comp. Appl. Math., vol. 162, pp. 213-229, 2004.

[11] A. J. Mayo and A. C. Antoulas, "A framework for the solution of the generalized realization problem," Linear Algebra \& Its App., vol. 425, pp. 634-662, 2007.

[12] T. C. Ionescu and A. Astolfi, "On moment matching with preservation of passivity and stability," in Proc. 49th IEEE Conf. on Decision and Control, 2010, pp. $6189-6194$.

[13] _ - "Families of moment matching based, structure preserving approximations for linear port Hamiltonian systems," Automatica, vol. 49, pp. 2424-2434, 2013.

[14] T. C. Ionescu, A. Astolfi, and P. Colaneri, "Families of moment matching based, low order approximations for linear systems," Systems \& Control Letters, vol. 64, pp. 47-56, 2014.

[15] G. Scarciotti and A. Astolfi, "Model reduction by moment matching for linear time-delay systems," in Proc. of the 19th IFAC World Congress, Cape Town, South Africa, August 2014.

[16] P. Kundur, Power systems stability and control. Mc Graw-Hill, 1994

[17] A. Giusto, R. Ortega, and A. Stankovic, "On transient stabilization of power systems: A power-shaping solution for structure-preserving models," in Proc. 45th IEEE Conf. on Decision and Control, 2006, pp. 4027-4031.

[18] E. Grimme, D. Sorensen, and P. V. Dooren, "Model reduction of state space systems via an implicitly restarted Lanczos method," Numerical algorithms, vol. 12, pp. 1-31, 1995.

[19] E. J. Grimme, "Krylov projection methods for model reduction," Ph.D. dissertation, ECE Dept., Univ. of Illinois, Urbana-Champaign, USA, 1997.

[20] A. Astolfi, "A note on model reduction by moment matching for nonlinear systems," in Proc. 8th IFAC Symposium on Nonlinear Control Systems, 2010, pp. 1244-1248.

[21] T. C. Ionescu and A. Astolfi, "Families of reduced order models that achieve nonlinear moment matching," in Proc. American Control Conference, 2013, pp. 5518-5523.

[22] A. Isidori, Nonlinear control systems, 3rd ed. New York: SpringerVerlag, 1995.
[23] A. Astolfi, "Model reduction by moment matching for nonlinear systems," in Proc. 47th IEEE Conf. on Decision and Control, 2008, pp. 4873-4878.

[24] A. Isidori and C. I. Byrnes, "Steady state response, separation principle and the output regulation of nonlinear systems," in Proc. of 28th IEEE Conf. Dec. Cont., 1989, pp. 2247-2251.

[25] A. Isidori and C. I. . Byrnes, "Output regulation of nonlinear systems," IEEE Trans. Autom. Contr., vol. 35, no. 2, pp. 131-140, 1990.

[26] J. Carr, Applications of center manifold theory. Springer Verlag, 1981.

[27] H. Nijmeijer and A. J. van der Schaft, Nonlinear dynamical control systems. New York: Springer-Verlag, 1990.

[28] H. Rodriguez, R. Ortega, and A. Astolfi, "Adaptive partial state feedback control of the DC-to-DC Ćuk converter," in Proc. of American Control Conf., 2005, pp. 5121-5126.

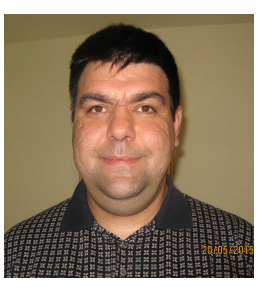

Tudor C. Ionescu was born in Bucharest, Romania in 1980. He received an M.Sc. in Systems and Control Engineering from the Politehnica University of Bucharest, Romania (2004) and the Ph.D. in Applied Mathematics from the University of Groningen, the Netherlands (2009). During his Ph.D. he has been a visiting researcher at SUPÉLEC, Paris, France (2005 and 2007) and at Nagoya University, Japan (2008) He was a Research Associate at Imperial College London, UK (2009-2013) and at the University of Sheffield, UK (2013-2015). During his post-doctoral years he has been awarded the Dutch Science Foundation (NWO) Visitor Grant at the University of Groningen, the Netherlands (2011 and 2015). Currently, he is a part-time Researcher at the "Gheorghe Mihoc - Caius Iacob" Institute for Mathematical Statistics and Applied Mathematics of the Romanian Academy, Bucharest, Romania. He is soon to be a full-time Assistant Professor at the Department of Automatic Control and Systems Engineering, Politehnica University of Bucharest, Romania. His research interests include modelling and control of nonlinear systems with focus on modelling and model order reduction with preservation of physical structure, as well as model reduction based finite-dimensional approximation of distributed-parameter systems.

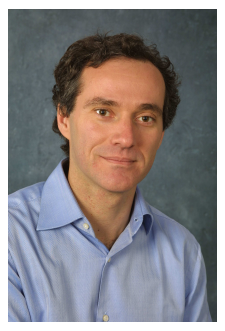

Alessandro Astolfi as born in Rome, Italy, in 1967. He graduated in Electrical Engineering from the University of Rome in 1991. In 1992 he joined ETHZurich where he obtained a M.Sc. in Information Theory in 1995 and the Ph.D. degree with Medal of Honor in 1995 with a thesis on discontinuous stabilization of nonholonomic systems. In 1996 he was awarded a Ph.D. from the University of Rome "La Sapienza" for his work on nonlinear robust control. Since 1996 he has been with the Electrical and Electronic Engineering Department of Imperial College London, London (UK), where he is currently Professor in Nonlinear Control Theory and Head of the Control and Power Group. From 1998 to 2003 he was also an Associate Professor at the Dept. of Electronics and Information of the Politecnico of Milano. Since 2005 he has also been a Professor at Dipartimento di Ingegneria Civile e Ingegneria Informatica, University of Rome Tor Vergata. He has been a visiting lecturer in "Nonlinear Control" in several universities, including ETH-Zurich (1995-1996); Terza University of Rome (1996); Rice University, Houston (1999); Kepler University, Linz (2000); SUPÉLEC, Paris (2001). His research interests are focused on mathematical control theory and control applications, with special emphasis for the problems of discontinuous stabilization, robust and adaptive control, observer design and model reduction. He is the author of more than 120 journal papers, of 30 book chapters and of over 240 papers in refereed conference proceedings. He is the recipient of the IEEE CSS A. Ruberti Young Researcher Prize (2007) and of the IEEE CSS George S. Axelby Outstanding Paper Award (2012). He is a "Distinguished Member" of the IEEE CSS. He is the author (with D. Karagiannis and R. Ortega) of the monograph "Nonlinear and Adaptive Control with Applications" (Springer-Verlag).

$\mathrm{He}$ is Associate Editor of Automatica and of the International Journal of Control, and Area Editor for the International Journal of Adaptive Control and Signal Processing. He is Senior Editor of the IEEE Trans. on Automatic Control and Editor-in-Chief of the European Journal of Control. He is the Chair of the IEEE CSS Conference Editorial Board. 